\title{
Health Care Resource Utilization and Costs Associated with Nonfatal Major Adverse Cardiovascular Events
}

\author{
Jennifer S. Korsnes, MS; Keith L. Davis, MA; Rinat Ariely, MSc, MBA; \\ Christopher F. Bell, MS; and Debanjali Mitra, MBA, MA
}

\begin{abstract}
BACKGROUND: Risk of recurrent cardiovascular events following an initial cardiovascular-related hospitalization remains high despite available interventions. Rates of cardiovascular events as well as associated health care resource utilization and costs are needed to assess the value of treatments.

OBJECTIVE: To quantify, in patients with previous hospitalization for acute coronary syndrome (ACS), rates of nonfatal major adverse cardiovascular events (MACE) and secondary coronary events (SCE), as well as health care utilization and costs associated with a first MACE.

METHODS: Administrative data from a large population of commercial managed care and managed Medicare enrollees in the United States were retrospectively analyzed. Patients with an ACS-related hospitalization from 2006 to 2011 were followed for 12 months to assess subsequent MACE and SCE rates. Patients were aged $\geq 18$ years at initial ACS hospitalization (the index episode) and had $\geq 12$ months of continuous health plan enrollment before and after the end of the index episode. Resource utilization and costs during a first MACE were assessed. Multivariable analyses were used to assess the associations between cardiovascular risk factors and the occurrence of a MACE, as well as the costs incurred during a first MACE.
\end{abstract}

RESULTS: Of 75,231 study patients identified, 3.3\% had a MACE and $8.3 \%$ had an SCE during the 12-month follow-up. Median time to first MACE and SCE from end of the index episode was 4.6 and 3.7 months, respectively. Mean MACE-related cost incurred during the first MACE was $\$ 19,642$. Logistic analyses showed that age and diabetes were associated with increased odds of a MACE, while index ACS episodes involving ST-elevation myocardial infarction were associated with reduced odds. Findings from generalized linear models indicated that statin use and age were associated with lower episode-related costs and that MACE occurrence within 3 months of ACS hospitalization was associated with increased episoderelated costs.

CONCLUSIONS: MACES and SCEs represent a common and costly burden in the year following ACS hospitalization. Our findings may inform future economic assessments of new therapies aimed at prevention of MACEs and SCEs.

J Manag Care Spec Pharm. 2015;21(6):443-50

Copyright @ 2015, Academy of Managed Care Pharmacy. All rights reserved.

\section{What is already known about this subject}

Despite available treatments, risk of recurrent cardiovascular events remains high, especially in high-risk populations.

Cardiovascular diseases represent some of the most costly chronic conditions, estimated to cost approximately $\$ 315.4$ billion in 2010 .

\section{What this study adds}

This study adds to what is known about the incidence and cost of major adverse cardiovascular events (MACE) and secondary coronary events (SCE) following hospitalization for acute coronary syndrome (ACS).

We found that $3.3 \%$ of patients had at least 1 MACE, and $8.3 \%$ had at least 1 SCE during the 12 months following an ACS hospitalization

Mean MACE-related costs incurred during the first MACE was $\$ 19,642$, and a MACE occurring within 3 months of an ACS hospitalization was associated with increased costs, whereas age at the time of ACS hospitalization and statin use were associated with decreased costs.

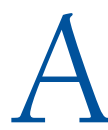
s the leading cause of death in the United States, cardiovascular diseases (CVDs) accounted for approximately $25 \%$ of all deaths in 2010. ${ }^{1}$ Two of the most common types of CVDs are myocardial infarction (MI) and stroke, which impose considerable clinical and economic burden on society. Annually, approximately 720,000 and 795,000 Americans have an MI or stroke, respectively, and the estimated total cost associated with CVDs was $\$ 315.4$ billion in 2010. ${ }^{1-3}$

Several types of interventions reduce the risk of CVDs, including pharmacological treatments (e.g., antihypertensives, antihyperlipidemics, and antiplatelets); medical devices (e.g., pacemakers, coronary stents, and implantable defibrillators); and surgical coronary revascularization procedures (e.g., coronary artery bypass graft and percutaneous coronary intervention). ${ }^{4}$ However, despite the treatments available, risk of recurrent cardiovascular events remains considerable. Previous trials have shown that even with medication, the risk of a recurrent cardiovascular event could exceed 20\% among high-risk populations (e.g., adults with coronary disease, other occlusive arterial disease, or diabetes). ${ }^{5,6}$ This high residual risk highlights the need for new treatments that further reduce the risk of cardiovascular events. ${ }^{7}$

To better understand the economic burden of CVDs and inform future cost and cost-effectiveness assessments of new therapies aimed at cardiovascular event prevention, this study aimed to quantify rates of nonfatal major adverse cardiovascular events (MACE) and secondary coronary events (SCE) 
in the 12 months following hospitalization for acute coronary syndrome (ACS) in a large commercially insured population. Among patients with at least 1 subsequent MACE, health care resource utilization, such as hospitalizations, procedures, and outpatient visits, and costs associated with the first MACE were assessed. Within the context of this study, MACE was defined by hospitalization for MI or stroke, and SCE was defined by hospitalization for heart failure, unstable angina, transient ischemic attack, limb amputation due to vascular causes, or coronary revascularization.

\section{Methods}

\section{Data Source}

Administrative claims data spanning January 1, 2006, to December 31, 2011, from a large population of commercial managed care and managed Medicare enrollees in the United States were retrospectively analyzed. These data were taken from the MarketScan Commercial Claims and Encounters (CCAE) and Medicare Supplemental Database provided by Truven Health Analytics. The database consists primarily of employer and health plan data containing patient demographics, periods of health plan enrollment, primary and secondary diagnoses, and detailed information about medical and drug utilization and costs. However, information on prescription medications dispensed via hospital pharmacies during inpatient stays was not captured. Patient mortality also was not captured. Because all data were pre-existing and de-identified, an authorized institutional review board, the RTI International Committee for the Protection of Human Subjects (FederalWide Assurance \#3331), granted approval for study conduct and exemption from requirement of patient-informed consent.

\section{Patient Selection}

Patients were selected for inclusion if they had a primary inpatient hospital discharge diagnosis of ACS (International Classification of Diseases, Ninth Revision, Clinical Modification [ICD-9-CM] codes 410.xx [acute myocardial infarction], 411.1 [intermediate coronary syndrome], 411.8x [other acute and subacute forms of ischemic heart disease]) between January 1, 2007, and December 31, 2010. The admission date of the first observed ACS hospitalization defined the index episode start date, and the date 30 days after the hospital discharge was considered the index episode end date. The 30-day extension beyond discharge from the index ACS hospital episode was applied to avoid misidentification of index episode-related resource use as recurring MACEs. The immediate date after completion of the index episode defined the follow-up start date, from which all outcomes were measured for 12 months thereafter. Patients were required to be at least aged 18 years at the index episode date and to have had medical and pharmacy benefits for at least 12 continuous months before the index episode (pre-index period) and after the end of the index episode (post-index period).

\section{Study Measures}

Patient characteristics documented in this study included age, sex, geographic region, and health plan type on the hospital admission date for the index episode. The presence of cardiovascular risk factors, including age of at least 60 years at index, pre-index diabetes requiring treatment, pre-index renal disease, and index diagnosis of ST-elevation MI (STEMI) versus non-ST-elevation MI (NSTEMI; Appendix A, available in online article), as well as index statin use and the Charlson Comorbidity Index (CCI) score, were assessed. ${ }^{8}$ We also flagged additional pre-index conditions (Appendix B, available in online article) that have been examined in previous clinical trials in similar populations (e.g., O'Donoghue et al. [2011] ${ }^{9}$ and White et al. [2014] ${ }^{10}$ which assessed the efficacy of darapladib in MACE prevention).

In addition to patient characteristics, outcomes of interest included the occurrence of MACEs and SCEs, as well as health care resource utilization and costs associated with first MACEs subsequent to the index ACS hospital episode. MACE was defined in this study as a composite of MI (ICD-9-CM diagnosis code 410.xx [acute myocardial infarction]) and acute ischemic stroke (ICD-9-CM diagnosis codes 433.xx [occlusion and stenosis of precerebral arteries], 434.xx [occlusion of cerebral arteries], and 436.xx [acute, but ill-defined, cerebrovascular disease]). Primary inpatient discharge diagnoses were used to identify the occurrence of a MACE. Nonprimary inpatient discharge diagnoses and outpatient diagnoses for MI and stroke were not used to avoid inclusion of claims likely related to older episodes or history of MACE. Because the study database does not provide complete mortality information, it was not possible to definitively delineate fatal versus nonfatal MACEs for study patients. However, given the study criterion requiring at least 12 months of plan enrollment after the index ACS episode (for provision of adequate follow-up for assessing first and subsequent MACE frequency and MACE costs), any MACE occurring in that time frame, except perhaps a MACE at the end of the 12-month follow-up, was by definition a nonfatal event.

SCE was defined in this study as a composite of hospitalization for heart failure (ICD-9-CM diagnosis code 428.xx), hospitalization for unstable angina (ICD-9-CM diagnosis code 413.xx [angina pectoris]), transient ischemic attack (ICD9-CM diagnosis code 435.x [transient cerebral ischemia]), limb amputation due to vascular causes (ICD-9-CM procedure code $84.0 x-84.1 x$ without any primary or secondary diagnoses for injury from the same confinement [ICD-9-CM diagnosis code 800.xx-999.xx (injury and poisoning]), and coronary revascularization, including percutaneous coronary intervention (ICD-9-CM procedure codes 36.0x, 00.66; Healthcare Procedure Coding System [HCPCS] codes G0290, G0291; and Current Procedural Terminology [CPT] codes 92973-92975, 92980, 92981, 91984, 92995-92998), and coronary artery bypass graft (ICD-9-CM procedure code 36.1x; HCPCS codes 


\section{FIGURE 1 Sample Selection Flowchart}

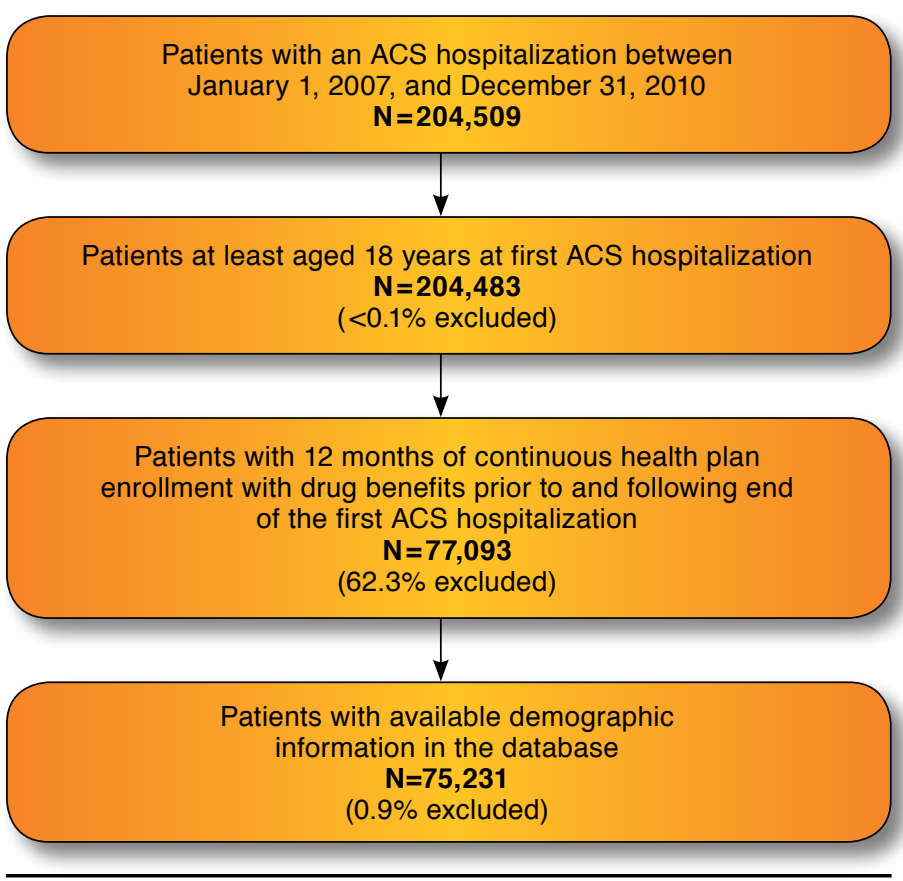

ACS = acute coronary syndrome.

S2205-S2209; CPT codes 33510-33536). Similar to MACEs, SCEs were assessed using primary inpatient discharge diagnoses to avoid capture of claims likely related to older episodes or history of SCEs.

The number and percentage of patients with at least 1 MACE or SCE, as well as those with each of the component conditions during the 12-month follow-up period after the end of the index episode, were reported. Among patients who experienced a MACE or an SCE, time to MACE and SCE were reported. Also among patients who experienced a MACE, resource use and costs associated with the first MACE were reported. A MACE was defined as the period beginning with the hospital admission date of the episode (i.e., MACE onset) until 30 days after the hospital discharge date. A MACE that was censored at the end of follow-up before 30 days after the discharge date was excluded from the cost analysis. The 30-day postdischarge extension of a MACE was imposed to allow for sufficient observation of event-related resource use and to avoid misattribution of these resources to another separate MACE.

For each patient with a MACE, health care utilization and associated costs were aggregated across all MACE-related encounters observed during the first MACE and were reported overall and by the major service setting in which they occurred (i.e., inpatient, emergency department, office visit, pharmacy, other outpatient, or ancillary, identified by place of service on each claim). For each service setting, the number and percent- age of patients with a visit and the mean (standard deviation [SD]) and median number of visits and costs during the first MACE were reported. The reported costs represented payments made by the submitting plan for services incurred and were adjusted for inflation to 2012 U.S. dollars using the medical care component of the Consumer Price Index. Encounters during the first MACE with primary or nonprimary diagnoses or procedures for a MACE or an SCE or an outpatient prescription for a CVD-related therapy (identified using generic drug names and database-specific therapy class field; see Appendix $\mathrm{C}$, available in online article) were defined as MACE related.

\section{Analytic Strategies}

All analyses were conducted using SAS version 9.3 statistical software (SAS Institute, Inc., Cary, NC). Descriptive analyses were conducted for all measures and included means, SDs, and medians for continuous variables and frequency distributions for categorical variables. Logistic regressions were used to assess the relationship between cardiovascular risk factors and the occurrence of MACEs. Generalized linear models (GLMs) with a log-link function and a gamma distribution were estimated to assess the relationship between cardiovascular risk factors and MACE-related costs incurred during the first MACE for the subset of patients with a MACE. The risk factors assessed included at least aged 60 years at index, pre-index diabetes requiring treatment, pre-index renal disease, index STEMI event (compared with index NSTEMI event), and index statin use in the context of logistic regressions and GLMs, as well as having a MACE within 3 months of index (compared with after 3 months) for GLMs. These multivariable models additionally controlled for baseline age, sex, geographic (census) region, health plan type, presence of pre-index flagged conditions, preindex CCI score, and statin use prior to post-index follow-up. Significance was established at the 0.05 alpha level.

\section{Results}

A total of 204,509 patients with an ACS-related hospitalization were initially identified from the study database (Figure 1). After application of the study inclusion criteria, 75,231 ACS patients remained in the study sample. The requirement of at least 12 months of continuous health plan enrollment before and after the index date was the primary reason for sample attrition. All selection criteria combined eliminated $63.2 \%$ of the starting sample, resulting in a study sample of 75,231 .

Mean [SD] age of the study sample was 64.4 [13.4] years (Table 1). Patients of working age (18-64 years) represented more than half $(57.0 \%)$ of the patients, and persons aged 65 years and older comprised $43.0 \%$ of the sample. Approximately two-thirds of ACS patients were male. Based on the geographic dispersion of employers and health plans captured in the CCAE database, the Midwest and South regions had the largest representation in the study sample $(34.9 \%$ and $36.1 \%$, 


\begin{tabular}{|c|c|c|}
\hline \multirow{2}{*}{$\begin{array}{l}\text { Patient Characteristics } \\
\text { Age (mean }[S D], \text { median) }\end{array}$} & \multicolumn{2}{|c|}{$\mathrm{N}=75,231$} \\
\hline & $64.4[13.4]$ & 62.0 \\
\hline & $\%$ & $\mathbf{n}$ \\
\hline \multicolumn{3}{|l|}{ Age group } \\
\hline $18-34$ & 0.6 & 455 \\
\hline $35-44$ & 4.7 & 3,535 \\
\hline $45-54$ & 18.6 & 14,015 \\
\hline $55-59$ & 15.7 & 11,838 \\
\hline $60-64$ & 17.3 & 13,043 \\
\hline $65+$ & 43.0 & 32,345 \\
\hline Male sex & 65.8 & 49,489 \\
\hline \multicolumn{3}{|l|}{ Geographic region } \\
\hline Northeast & 12.4 & 9,330 \\
\hline Midwest & 34.9 & 26,224 \\
\hline South & 36.1 & 27,156 \\
\hline West & 16.0 & 12,002 \\
\hline Unknown & 0.7 & 519 \\
\hline Current statin use ${ }^{a}$ & 73.1 & 55,018 \\
\hline \multicolumn{3}{|l|}{ Index ACS diagnosis } \\
\hline STEMI & 22.4 & 16,887 \\
\hline NSTEMI & 38.8 & 29,173 \\
\hline Unstable angina & 38.8 & 29,171 \\
\hline \multicolumn{3}{|c|}{ Preindex comorbid conditions of interest } \\
\hline Diabetes requiring treatment & 20.6 & 15,468 \\
\hline Renal disease & 0.1 & 49 \\
\hline \multicolumn{3}{|l|}{ Pre-index CCI score } \\
\hline 0 & 29.3 & 22,016 \\
\hline 1 & 25.2 & 18,989 \\
\hline 2 & 16.8 & 12,668 \\
\hline 3 or greater & 28.7 & 21,558 \\
\hline Mean [SD], median & $1.9[2.2]$ & 1.0 \\
\hline \multicolumn{3}{|c|}{$\begin{array}{l}\text { aStatin prescription that overlaps with the end of the index episode or the start } \\
\text { the follow-up period. } \\
\text { ACS=acute coronary syndrome; CCI=Charlson Comorbidity Index; } \\
\text { NSTEMI=non-ST-elevation myocardial infarction; SD=standard deviation; } \\
\text { STEMI =ST-elevation myocardial infarction. }\end{array}$} \\
\hline
\end{tabular}

respectively). Compared with the general U.S. population, the Northeast was underrepresented in this study (12.4\%). A substantial proportion of patients were receiving statin treatment at the index episode date (73.1\%). Patients had an index diagnosis of NSTEMI as frequently as unstable angina (38.8\% of patients, each), whereas an index diagnosis of STEMI was less frequently observed (22.4\% of patients). Examining specific comorbid conditions and clinical events of interest, we observed that diabetes requiring treatment was relatively common $(20.6 \%$ of patients), whereas renal disease was not $(0.1 \%$ of patients). The mean [SD] and median CCI score, assessed during 12 months prior to the index ACS hospital admission date, were relatively low at 1.9 [2.2] and 1.0, respectively, based upon previously established indices. ${ }^{11}$

\begin{tabular}{|c|c|c|}
\hline \multicolumn{3}{|c|}{$\begin{array}{c}\text { TABLE 2 Major Adverse Cardiovascular Events } \\
\text { and Secondary Coronary Events During } \\
\text { the 12-Month Follow-up Period }\end{array}$} \\
\hline \multirow{2}{*}{$\begin{array}{l}\text { Event } \\
\text { Patients with Event }\end{array}$} & \multicolumn{2}{|c|}{$\mathrm{N}=75,231$} \\
\hline & $\%$ & $\mathbf{n}$ \\
\hline \multicolumn{3}{|l|}{$\overline{\text { MACE }^{a}}$} \\
\hline First MACE & 3.3 & 2,503 \\
\hline Second MACE & 0.2 & 137 \\
\hline Third MACE & $<0.1$ & 9 \\
\hline \multicolumn{3}{|l|}{ Type of MACE (not mutually exclusive) } \\
\hline MI & 2.5 & 1,880 \\
\hline Stroke & 0.9 & 642 \\
\hline \multicolumn{3}{|l|}{ SCE $^{a}$} \\
\hline First SCE & 8.3 & 6,259 \\
\hline Second SCE & 0.9 & 688 \\
\hline Third SCE & 0.1 & 107 \\
\hline \multicolumn{3}{|l|}{ Type of SCE (not mutually exclusive) } \\
\hline Heart failure & 2.7 & 2,025 \\
\hline Unstable angina & 0.1 & 62 \\
\hline Transient ischemic attack & 0.2 & 188 \\
\hline Limb amputation due to vascular causes & 0.1 & 85 \\
\hline Coronary revascularization & 5.5 & 4,129 \\
\hline Days to Event from End of Prior Episode ${ }^{b}$ & Mean $[S D]$ & Median \\
\hline \multicolumn{3}{|l|}{ MACE } \\
\hline First MACE & 149.8 [93.9] & 139.0 \\
\hline Second MACE & $92.4[66.1]$ & 78.0 \\
\hline Third MACE & $50.6[40.9]$ & 44.0 \\
\hline \multicolumn{3}{|l|}{ SCE } \\
\hline First SCE & $129.1[94.0]$ & 112.0 \\
\hline Second SCE & $90.0[64.7]$ & 79.0 \\
\hline Third SCE & $59.3[47.6]$ & 48.0 \\
\hline Timing of First MACE Among Those with a MACE & $\%$ & $\mathbf{n}$ \\
\hline Within 3 months following index episode & 33.4 & 835 \\
\hline After 3 months following index episode & 66.6 & 1,668 \\
\hline \multicolumn{3}{|c|}{$\begin{array}{l}\text { Each event type is counted once per episode; however, multiple event types are } \\
\text { allowed per episode. } \\
\text { bEach episode is given } 30 \text { days from hospital discharge. } \\
\text { MACE = major adverse cardiovascular event; } M I=\text { myocardial infarction; } \\
\text { SCE = secondary coronary event; } S D=\text { standard deviation. }\end{array}$} \\
\hline
\end{tabular}

Overall, 2,503 ACS patients (3.3\%) had at least 1 MACE during the 12-month follow-up period (Table 2). However, the study inclusion criteria requiring a minimum 12-month follow-up period eliminated fatal MACEs, except for those experienced at the end of the follow-up period. Furthermore, mortality was not reported in the MarketScan CCAE database and hence not captured in this analysis. Among patients with at least 1 follow-up MACE, MI was the most common event type (1,880 patients: $75.1 \%$ of patients with MACEs, $2.5 \%$ of patients overall). Stroke was a relatively uncommon MACE in the post-ACS setting (642 patients, or $0.9 \%$ of patients) but was seen in a quarter (25.6\%) of all patients with MACEs. Among patients with a follow-up MACE, mean [SD] and median times to the first MACE from the end of the index episode were 149.8 
Health Care Resource Utilization and Costs Associated with Nonfatal Major Adverse Cardiovascular Events

TABLE 3 Logistic Regressions Evaluating Association Between Occurrence of MACEs and Measures of Interest

\begin{tabular}{|c|c|c|c|c|c|}
\hline Measures of Interest, Each from a Separate Modela & Odds Ratio & $95 \%$ CI & $P$ Value ${ }^{b}$ & Sample Size & $\begin{array}{l}\text { Number } \\
\text { of Cases }\end{array}$ \\
\hline At least aged 60 years at index $($ reference $=$ under 60$)$ & 1.233 & $(1.119-1.359)$ & $<0.001$ & 75,231 & 2,503 \\
\hline Pre-index diabetes requiring treatment (reference $=$ no diabetes) & 1.476 & $(1.340-1.625)$ & $<0.001$ & 75,231 & 2,503 \\
\hline Pre-index renal disease (reference $=$ no renal disease $)$ & 0.764 & $(0.185-3.156)$ & 0.710 & 75,231 & 2,503 \\
\hline Statin use at end of index episode (reference = no statin use) & 0.865 & $(0.793-0.943)$ & 0.001 & 75,231 & 2,503 \\
\hline STEMI index MI event (reference = NSTEMI) & 0.782 & $(0.698-0.875)$ & $<0.001$ & 46,060 & 1,610 \\
\hline
\end{tabular}

aEach model additionally controls for baseline age, sex, geographic (census) region, health plan type, presence of pre-index flagged conditions, pre-index CCI score, and statin use prior to post-index follow-up unless collinear with independent variable of interest.

${ }^{b}$ Not adjusted for multiple comparisons.

$C C I=$ Charlson Comorbidity Index; $C I=$ confidence interval; $M A C E=$ major adverse cardiovascular event; $M I=$ myocardial infarction; NSTEMI=non-ST-elevation myocardial infarction; STEMI=ST-elevation myocardial infarction.

[93.9] and 139.0 days, respectively. Approximately one-third of patients with at least 1 MACE experienced the event within 3 months after the index episode. Among patients with at least 1 MACE, 137 patients (5.5\% of those with an event) experienced a second event during the 12-month follow-up period. Mean [SD] and median times to the second MACE from the end of the prior MACE were 92.4 [66.1] and 78.0 days for patients with a second event, respectively. Only 9 patients experienced a third MACE during the 12-month follow-up period.

Overall, 6,259 patients (8.3\%) had at least 1 SCE during the 12-month follow-up period (Table 2). Among patients with at least 1 follow-up SCE, the most common type was coronary revascularization, observed in $66.0 \%$ of patients with an SCE and $5.5 \%$ of patients overall. The next most common SCE was heart failure, observed in $32.4 \%$ of patients with an SCE and $2.7 \%$ of patients overall. Unstable angina or noncoronary ischemic events, as well as limb amputations due to vascular causes, were infrequently observed SCEs.

From the logistic regression results (Table 3), being aged 60 years or older at the index episode date was associated with a 23.3\% increase in the odds of a MACE. Pre-index diabetes was associated with a $47.6 \%$ increase in the odds of experiencing a MACE. For patients whose index ACS episode involved an MI, having a STEMI diagnosis was associated with a $21.8 \%$ reduction in the odds of a MACE as compared with an NSTEMI diagnosis. Statin use at the end of the index ACS episode was associated with a $13.5 \%$ reduction in the odds of a MACE. Since only 49 patients had renal disease in the pre-index period, there was virtually no power to assess its relationship with events.

Among study patients with at least 1 MACE during the 12-month follow-up period, by definition, all had an inpatient hospital admission. Mean [SD] and median costs of $\$ 18,576$ $[\$ 38,027]$ and $\$ 5,973$, respectively, were observed for all MACE-related inpatient care during the first MACE (Table 4). Patients had an average of 1 MACE-related hospital admission during the first MACE. The majority of patients with MACEs (82.3\%) had a CVD-related outpatient prescription, with mean [SD] and median outpatient prescription costs during the first
MACE of \$228 [\$294] and \$146 per patient, respectively. We observed less than a quarter of patients visiting the emergency department or a physician's office during the first MACE for a MACE-related reason. Mean [SD] and median total episoderelated costs per patient for the first MACE were $\$ 19,642$ $[\$ 38,690]$ and $\$ 6,563$, respectively. During the first MACE, we found that MI-related costs accounted for $85.0 \%$ of total mean episode costs.

The multivariable GLM results (Table 5) indicate that being aged 60 years or older at the index episode date was associated with significantly lower cost $(63.4 \%$ lower) of the first MACE episode. Having a MACE within the first 3 months of follow-up was associated with a significant $35.5 \%$ increase in episode costs. Statin use at the end of the index episode was associated with a significant $9.2 \%$ reduction in the cost of the first MACE. We observed no significant association between episode costs and pre-index diabetes, pre-index renal disease, or index STEMI (for those with an index MI event).

\section{Discussion}

In this large-scale observational study, we assessed rates and costs of nonfatal MACEs in patients with a prior history (within the preceding year) of an ACS-related hospitalization to address current literature gaps and to provide supporting information for future economic assessments of therapies aimed at MACE prevention. A review of published reports also assessing population-based cardiovascular event rates may provide additional context to our findings. One recent claims-based study by Chapman et al. (2011) found an overall cardiovascular hospitalization event rate (MACEs and SCEs combined, defined by MI, heart failure, unstable angina, angina pectoris, other ischemic heart disease, ischemic stroke, transient ischemic attack, and peripheral vascular disease) of $16.1 \%$ over a variable follow-up period of up to 3 years after a previous cardiovascularrelated hospitalization..$^{12}$ Although the Chapman et al. study did not assess specific event rates separately, the reported composite rate of $16.1 \%$ falls moderately above the range of event rates we observed for MACEs and SCEs combined (11.6\%) during 12 


\begin{tabular}{|c|c|c|}
\hline \multicolumn{3}{|c|}{$\begin{array}{ll}\text { TABLE } 4 & \text { MACE-Related }^{a} \text { Health Care } \\
& \text { Resource Utilization and Costs } \\
& \text { Incurred During the First MACE }\end{array}$} \\
\hline Resources & $\mathrm{N}=2$ & \\
\hline Patients with At Least 1 Service & $\%$ & n \\
\hline Hospitalizations & 100.0 & 2,503 \\
\hline Emergency department & 18.1 & 452 \\
\hline Physician's office & 22.0 & 550 \\
\hline Pharmacy (outpatient) & 82.3 & 2,059 \\
\hline Ancillary outpatient & 47.9 & 1,200 \\
\hline MI-related & 76.6 & 1,918 \\
\hline Stroke-related & 31.1 & 778 \\
\hline Total & 100.0 & 2,503 \\
\hline Number of Services & Mean [SD] & Median \\
\hline Hospitalizations & $1.1 \quad[0.4]$ & 1.0 \\
\hline Emergency department & $0.2 \quad[0.4]$ & 0.0 \\
\hline Physician's office & $0.3 \quad[0.8]$ & 0.0 \\
\hline Pharmacy (outpatient) & $\begin{array}{ll}3.7 & {[2.9]} \\
\end{array}$ & 3.0 \\
\hline Ancillary outpatient & $1.3 \quad[2.6]$ & 0.0 \\
\hline MI-related & $\begin{array}{ll}6.6 & {[7.7]} \\
\end{array}$ & 5.0 \\
\hline Stroke-related & $2.5 \quad[5.4]$ & 0.0 \\
\hline Total & $\begin{array}{ll}6.6 & {[4.1]} \\
\end{array}$ & 6.0 \\
\hline Costs per Patient & Mean [SD] (\$) & Median (\$) \\
\hline Hospitalizations & $18,576 \quad[38,027]$ & 5,973 \\
\hline Emergency department & $34 \quad[138]$ & 0 \\
\hline Physician's office & $57 \quad[1,234]$ & 0 \\
\hline Pharmacy (outpatient) & [294] & 146 \\
\hline Ancillary outpatient & $748 \quad[3,917]$ & 0 \\
\hline MI-related & $16,698 \quad[38,016]$ & 2,099 \\
\hline Stroke-related & $5,043 \quad[25,689]$ & 0 \\
\hline Total & $19,642 \quad[38,690]$ & 6,563 \\
\hline
\end{tabular}

aMACE-related services were identified as those with a MACE diagnosis, an SCE diagnosis or procedure, or a CVD drug prescription.

${ }^{b}$ Each episode was given 30 days from hospital discharge.

$C V D=$ cardiovascular disease; $M A C E=$ major adverse cardiovascular event; $M I=$ myocardial infarction; $S C E=$ secondary coronary event; $S D=$ standard deviation.

months of follow-up. The difference observed may be due to the inclusion of fatal events, peripheral vascular disease overall (rather than limb amputations due to peripheral vascular disease), and angina pectoris (in addition to unstable angina), as well as a longer follow-up period.

Additional studies have assessed cardiovascular event rates in patients with prior cardiac history using large-scale, prospective patient registries. A notable example is the Reduction of Atherothrombosis for Continued Health (REACH) Registry, which is a contemporary international outpatient study of patients with either stable symptomatic vascular disease (coronary heart disease, stroke, transient ischemic attack, or peripheral artery disease) or multiple risk factors for vascular disease (e.g., hypertension, diabetes, hyperlipidemia, carotid stenosis, and smoking). ${ }^{13}$ One study based on REACH data by Mahoney et al. (2008) reported 1-year cardiovascular event rates for patients in the United States with a prior history of coronary heart disease and other high-risk vascular conditions. ${ }^{14}$ In the Mahoney et al. study, the overall percentage of patients with at least 1 composite cardiovascular event (i.e., cardiovascular death, MI, or stroke) after 1 year of follow-up was 4\%. Although our study could not assess fatal events, our estimate of the proportion of patients with at least $1 \mathrm{MI}(2.5 \%)$ and 1 stroke $(0.9 \%)$, when combined, approached the 1-year composite rate reported by Mahoney et al.

As confirmed in other studies, ${ }^{15,16}$ older age and diabetes were associated with increased risk of a MACE, whereas statin use was associated with a decreased risk of a MACE. ${ }^{17}$ We also found that patients with a STEMI index event were at somewhat lower risk of a MACE than patients with an NSTEMI index event, among the subset of patients who had 1 of these types of index events, although the mechanism behind this association is unclear and warrants further exploration. One previous study in a Korean population also found a lower 12-month risk of nonfatal MI and coronary artery bypass graft following a previous STEMI than following a previous NSTEMI. ${ }^{18}$ One possible explanation for lower subsequent MACE risk in STEMI patients is that these patients may have received more extensive disease intervention (coronary stenting, catheterization, revascularization, and other procedures often emergently needed in STEMI cases) as compared with NSTEMI patients during the index ACS hospitalization. If true, this finding may suggest that more aggressive intervention for NSTEMI may decrease subsequent MACE risk.

The large mean health care costs observed for the first MACE during the 12-month follow-up period were consistent with previous findings from similar studies. Closely matching estimations of cardiovascular events were reported by Chapman et al., with an average hospitalization cost for a subsequent cardiovascular event following an index cardiovascular event of $\$ 17,709 . .^{12}$ These estimates were slightly lower than those observed in this study $(\$ 18,576)$. The differences observed between these studies may be attributed to our inclusion of the 30-day episode extension following discharge from the hospital versus their restriction to the inpatient stay associated with the event, as well as to different conditions and procedures designated as disease related. Based upon the multivariable analyses, we observed a significant reduction in the cost of the first MACE among patients aged 60 years or older, possibly due to differing health plan payment representation within the database among retired patients.

Since previous research has shown an association between statin use and reduced health care costs, ${ }^{19}$ we also found that patients with statin use at the end of the index episode had reduced first MACE costs, which may indicate a reduction in cardiovascular complications associated with statin use. ${ }^{20}$ Furthermore, the increased first MACE costs found among patients with an early MACE (within 3 months of the index ACS hospital discharge) warrant further exploration, with an emphasis on characterizing the event severity and treatment profile of the index ACS and subsequent MACE episodes. 
TABLE 5 Generalized Linear Models Evaluating Association Between MACE-Related ${ }^{a}$ Health Care Costs of First MACE and Measures of Interest Among Patients with a MACE

\begin{tabular}{|c|c|c|c|c|}
\hline Measures of Interest, Each from a Separate Model ${ }^{b}$ & Rate Ratio ${ }^{c}$ & $95 \% \mathrm{CI}$ & $P$ Value & Sample Size \\
\hline At least aged 60 years at index $($ reference $=$ under 60$)$ & 0.366 & $(0.328-0.408)$ & $<0.001$ & 2,503 \\
\hline Pre-index diabetes (reference $=$ no diabetes) & 0.960 & $(0.866-1.064)$ & 0.437 & 2,503 \\
\hline Pre-index renal disease (reference $=$ no renal disease) & 2.684 & $(0.588-12.247)$ & 0.203 & 2,503 \\
\hline Statin use at end of index episode (reference = no statin use) & 0.908 & $(0.828-0.996)$ & 0.041 & 2,503 \\
\hline Early MACE within 3 months (reference=after 3 months) & 1.355 & $(1.237-1.484)$ & $<0.001$ & 2,503 \\
\hline STEMI index MI event (reference = NSTEMI) & 0.994 & $(0.876-1.129)$ & 0.931 & 1,610 \\
\hline \multicolumn{5}{|c|}{ 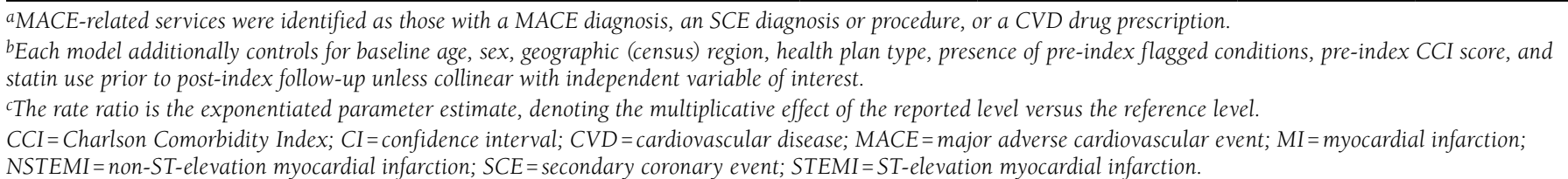 } \\
\hline
\end{tabular}

A study on the use of secondary prevention drug therapy among ACS patients suggests that patients are not commonly prescribed guideline medication therapies during the first 3 months following an ACS hospitalization, which may lead to more complicated cardiovascular events during this time..$^{21}$

\section{Limitations}

Our study was subject to several limitations that should be considered in the interpretation of our findings. First, patients were identified and costs were designated on the basis of ICD9-CM diagnosis codes and HCPCS, CPT, and ICD-9-CM procedure codes, with no access to patient charts for confirmation of events. The extent to which coding errors may have led to misclassification of patients is unclear, but it is likely small. ${ }^{22,23}$ Second, due to the lack of clinical and comprehensive mortality information in the MarketScan CCAE database, we could not further validate the occurrence of outcomes of interest and could not include death due to CVD as a component of MACEs. Detection of MACEs from primary discharge diagnoses and the designation of 30 days after MACE discharge as the episode duration may have led to an underestimation of the number of outcomes observed during the follow-up period. Third, due to our requirement of continuous health plan enrollment for 12 months following the index episode, we may have introduced selection bias toward healthier patients in these data. On a related note, due to the requirement of a 12-month pre-index period, we cannot be certain that the index ACS episode was a patient's first experience of an ACS episode. Fourth, treatment assessment was based upon outpatient prescription claims data, which reflect medications prescribed, not necessarily medications taken. Fifth, the MarketScan CCAE database does not provide details on prescription drug use during hospitalization, thereby underestimating the use of pharmacotherapy. Sixth, we assessed only the direct medical costs associated with cardiovascular episodes and did not have data to examine the overall burden of these conditions to include indirect costs (e.g., lost workplace productivity and caregiver burden). Finally, this study was limited to managed care enrollees; thus, the results may not be generalizable to the entire U.S. population or to individuals covered by other federal insurance programs (e.g., Medicaid or Veterans Affairs). The Northeast's underrepresentation in our study sample further reduces generalizability; to the extent that per capita health care costs are higher in the Northeast as compared with other regions, our overall cost estimates for MACEs may be somewhat conservative.

\section{Conclusions}

Findings from this study provide current information on the characteristics, event rates, and episode-based health care resource use and costs of MACEs in a commercial population with previous history of ACS. The recurrence of cardiovascular events in the year following hospitalization for ACS, including MACEs and SCEs, represents a common and costly burden for patients in the United States. The data presented here may be useful in future evaluations of the cost-effectiveness of new cardiovascular treatments.

\section{Authors}

JENNIFER S. KORSNES, MS, is Associate Director, Health Economics, and KEITH L. DAVIS, MA, is Senior Director, Health Economics, RTI Health Solutions, Research Triangle Park, North Carolina. RINAT ARIELY, MSc, MBA, is Director, Global Health Outcomes, GlaxoSmithKline, King of Prussia, Pennsylvania; CHRISTOPHER F. BELL, MS, is Director, Global Health Outcomes, GlaxoSmithKline, Research Triangle Park, North Carolina; and DEBANJALI MITRA, MBA, MA, is Director, Outcomes and Evidence-Oncology, Pfizer, New York, New York.

AUTHOR CORRESPONDENCE: Jennifer S. Korsnes, MS, Associate Director, Health Economics, RTI Health Solutions, 200 Park Offices Dr., Research Triangle Park, NC 27709.

Tel.: 919.597.5121; Fax: 919.541.7222; E-mail: jkorsnes@rti.org. 


\section{DISCLOSURES}

This study was funded by GlaxoSmithKline, and authors are either employees of GlaxoSmithKline or received consulting fees from GlaxoSmithKline. Korsnes, Davis, and Mitra (and/or their institutions) received research funding from GlaxoSmithKline for this research study. Ariely and Bell are employees of GlaxoSmithKline group of companies.

Study concept and design were contributed by Ariely, Bell, and Mitra, along with Korsnes and Davis. Korsnes was primarily responsible for data collection, assisted by the rest of the authors. Analysis was performed by Korsnes, Davis, Ariely, and Bell, assisted by Mitra. The manuscript was written and revised by Korsnes, Davis, and Ariely, along with Bell and assisted by Mitra.

\section{REFERENCES}

1. Centers for Disease Control and Prevention. Heart disease facts. 2014. Available at: http://www.cdc.gov/heartdisease/facts.htm. Accessed May 5, 2015.

2. Centers for Disease Control and Prevention. Stroke facts. 2014. Available at: http://www.cdc.gov/stroke/facts.htm. Accessed May 5, 2015.

3. Go AS, Mozaffarian D, Roger VL, et al.; American Heart Association Statistics Committee and Stroke Statistics Subcommittee. Heart disease and stroke statistics-2014 update: a report from the American Heart Association. Circulation. 2014;129(3):e28-e292.

4. World Health Organization. The atlas of heart disease and stroke. Available at: http://www.who.int/cardiovascular_diseases/resources/atlas/en/. Accessed May 5, 2015.

5. Cannon CP, Braunwald E, McCabe $\mathrm{CH}$, et al.; Pravastatin or Atorvastatin Evaluation, Infection, Therapy-Thrombolysis in Myocardial Infarction 22 Investigators. Intensive versus moderate lipid lowering with statins after acute coronary syndromes. N Engl J Med. 2004;350(15):1495-504.

6. Heart Protection Study Collaborative Group. MRC/BHF heart protection study of cholesterol lowering with simvastatin in 20,536 high-risk individuals: a randomized placebo-controlled trial. Lancet. 2002;360(9326):7-22.

7. Bui QT, Wilensky RL. Darapladib. Expert Opin Investig Drugs. 2010;19(1):161-68

8. Charlson ME, Charlson RE, Peterson JC, Marinopoulos SS, Briggs WM, Hollenberg JP. The Charlson Comorbidity Index is adapted to predict costs of chronic disease in primary care patients. J Clin Epidemiol. 2008;61(12):1234-40.

9. O'Donoghue ML, Braunwald E, White HD, et al. Study design and rationale for the Stabilization of Plaques Using Darapladib-Thrombolysis in Myocardial Infarction (SOLID-TIMI 52) trial in patients after an acute coronary syndrome. Am Heart J. 2011;162(4):613-19.

10. White HD, Held C, Steward R, et al.; STABILITY Investigators. Darapladib for preventing ischemic events in stable coronary heart disease. N Engl J Med. 2014;370(18):1702-11.
11. Murray SB, Bates DW, Ngo L, Ufberg JW, Shapiro NI. Charlson Index is associated with one-year mortality in emergency department patients with suspected infection. Acad Emerg Med. 2006;13(5):530-36.

12. Chapman RH, Liu LZ, Girase PG, Straka RJ. Determining initial and follow-up costs of cardiovascular events in a US managed care population. BMC Cardiovasc Disord. 2011;11:11.

13. Ohman EM, Bhatt DL, Steg PG, et al.; REACH Registry Investigators. The REduction of Atherothrombosis for Continued Health (REACH) registry: an international, prospective, observational investigation in subjects at risk for atherothrombotic events-study design. Am Heart J. 2006;151(4):786. 14. Mahoney EM, Wang K, Cohen DJ, et al.; REACH Registry Investigators One-year costs in patients with a history of or at risk for atherothrombosis in the United States. Circ Cardiovasc Qual Outcomes. 2008;1(1):38-45.

15. Wald NJ, Simmonds M, Morris JK. Screening for future cardiovascular disease using age alone compared with multiple risk factors and age. PLoS One. 2011;6(5):e18742.

16. Yusuf S, Hawken S, Ôunpuu S, et al.; INTERHEART Study Investigators. Effect of potentially modifiable risk factors associated with myocardial infarction in 52 countries (the INTERHEART study): case-control study. Lancet. 2004;364(9438):937-52.

17. LaRosa JC, He J, Vupputuri S. Effect of statins on risk of coronary disease: a meta-analysis of randomized controlled trials. JAMA. 1999;282(24):2340-46.

18. Sim DS, Kim JH, Jeong MH. Differences in clinical outcomes between patients with ST-elevation versus non-ST-elevation acute myocardial infarction in Korea. Korean Circ J. 2009;39(8):297-303.

19. Zhao Y, Zabriski S, Bertram C. Association between statin adherence level, health care costs, and utilization. J Manag Care Pharm. 2014;20(7): 703-13. Available at: http://www.amcp.org/JMCP/2014/July/18272/1033. html.

20. O'Neil-Callahan K, Katsimaglis G, Tepper MR, et al. Statins decrease perioperative cardiac complications in patients undergoing noncardiac vascular surgery: the Statins for Risk Reduction in Surgery (StaRRS) study. J Am Coll Cardiol. 2005;45(3):336-42.

21. Lee HY, Cooke CE, Robertson TA. Use of secondary prevention drug therapy in patients with acute coronary syndrome after hospital discharge. J Manag Care Pharm. 2008;14(3):271-80. Available at: http://www.amcp.org/ data/jmcp/JMCPMaga_April08_271-280.pdf.

22. Losina E, Barrett J, Baron JA, Katz JN. Accuracy of medicare claims data for rheumatologic diagnoses in total hip replacement recipients. J Clin Epidemiol. 2003;56(6):515-19.

23. Newcomer R, Clay T, Luxenberg JS, Miller RH. Misclassification and selection bias when identifying Alzheimer's disease solely from Medicare claims records. J Am Geriatr Soc. 1999;47(2):215-19. 


\section{APPENDIX A Cardiovascular Risk Factors}

Risk Factor

\section{At least aged 60 years at index}

Pre-index diagnosis of diabetes requiring treatment

Pre-index renal disease (diagnosis of stage 3 or higher chronic kidney

disease, kidney transplant, or nephrectomy)
Identification Codes and Methods

ICD-9-CM diagnosis codes: 250.xx with prescription for antidiabetic drug ICD-9-CM diagnosis codes: 584.xx, 585.3-585.6, 586.xx, 403.01, 403.11, 403.91, 404.02, 404.03, 404.12, 404.13, 404.92, 404.93

HCPCS codes: 50220, 50225, 50230, 50234, 50236, 50240, 50340, 50360, $50365,50370,50543,50545,50546,50548$

Index myocardial infarction

\begin{tabular}{l|l}
\hline STEMI & ICD-9-CM diagnosis codes: 410.0x-410.6x, 410.8x \\
\hline NSTEMI & ICD-9-CM diagnosis codes: 410.7x \\
\hline
\end{tabular}

HCPCS = Healthcare Common Procedure Coding System; ICD-9-CM = International Classification of Diseases, Ninth Revision, Clinical Modification; NSTEMI = non-STelevation myocardial infarction; STEMI = ST-elevation myocardial infarction.

\section{APPENDIX B Flagged Conditions of Interest}

\section{Condition}

Liver disease, cirrhosis, or disorders of the biliary tract

Stage 4 or higher chronic kidney disease, kidney transplant, or nephrectomy

ICD-9-CM diagnosis code: 570-573.x, 576.x, 070.22, 070.23, 070.32, 070.33, $070.44,070.54,070.6,070.9, \mathrm{~V} 42.7$

ICD-9-CM diagnosis code: 584.xx, 585.4-585.6, 586.xx, 403.01, 403.11, 403.91, 404.02, 404.03, 404.12, 404.13, 404.92, 404.93, V42.0

HCPCS code: 50220, 50225, 50230, 50234, 50236, 50240, 50340, 50360, $50365,50370,50543,50545,50546,50548$

Hospitalization or emergency department visit due to heart failure, hypertension, asthma, or chronic obstructive pulmonary disease

ICD-9-CM diagnosis code: 428.xx, 401.x, 997.91, 491.xx-494.x, 496 398.91, 402.01, 402.11, 402.91, 404.01, 404.03, 404.11, 404.13, 404.91, 404.93, 425.4, 425.5, 425.7-425.9

Any life-threatening condition including HIV/AIDS and cancer (other than nonmelanoma skin cancer) and excluding vascular disease

Pregnancy

Anaphylaxis

Alcohol abuse, drug abuse, or mental health disorders

ICD-9-CM diagnosis code: 042-044, 140.x-172.x, 174.x-208.xx, 238.6

ICD-9-CM diagnosis code: V22.x, V23.x, V24.0, V24.1, V27.x, V28.x ICD-9-CM diagnosis code: 995.0, 995.27, 995.3, 995.6x, 999.4x, V13.81 ICD-9-CM diagnosis code: 290.xx-301.xx, 303.xx-305.xx, 306.xx-319

HCPCS = Healthcare Common Procedure Coding System; HIV/AIDS = human immunodeficiency virus/acquired immunodeficiency syndrome; ICD-9-CM = International Classification of Diseases, Ninth Revision, Clinical Modification. 


\section{APPENDIX C Cardiovascular Disease-Related Therapies}

\begin{tabular}{|c|c|}
\hline Drug Class & Generic Names \\
\hline Alpha beta blockers & carvedilol, labetalol \\
\hline Angiotensin receptor blockers & candesartan, irbesartan, losartan, telmisartan, valsartan \\
\hline Angiotensin converting enzyme inhibitors & $\begin{array}{l}\text { benazepril, captopril, enalapril, fosinopril, lisinopril, moexipril, perindopril, quinapril, ramipril, } \\
\text { trandolapril }\end{array}$ \\
\hline Antiarrhythmic agents & amiodarone, flecainide, procainamide, sotalol \\
\hline Antihyperlipidemics & $\begin{array}{l}\text { colestilan, dextran, elastase, fenofibrate, fenofibric acid, gemfibrozil, niceritrol, nicomol, } \\
\text { polyenephosphatidyl, probucol, soy sterol }\end{array}$ \\
\hline Antihypertensives, hypotensives, and vasodilators & $\begin{array}{l}\text { alacepril, aliskiren, benazepril, benzylhydrochlorothiazide, budralazine, bunazosin, cadralazine, } \\
\text { candesartan cilexetil, captopril, carbazochrome, cilazapril hydrate, clonidine, delapril, doxazosin, } \\
\text { enalapril maleate, guanabenz, hydralazine, imidapril, irbesartan, lisinopril, losartan, methyldopa, } \\
\text { olmesartan medoxomil, perindopril erbumine, prazosin, quinapril, reserpine, sodium nitroprusside, } \\
\text { telmisartan, temocapril, terazosin, trandolapril, urapidil, valsartan }\end{array}$ \\
\hline Antithrombotics & $\begin{array}{l}\text { acenocoumarol, acetylsalicylate, acetylsalicylic, alteplase, carbasalate, eicosapentaenoic, eptifibatide, } \\
\text { ethyl icosapentate, fondaparinux, nadroparin, phenprocoumon, prasugrel, reteplase, streptokinase, } \\
\text { sulodexide, tenecteplase, tissue plasminogen activator, triflusal, urokinase }\end{array}$ \\
\hline Antithrombotics: anticoagulants & argatroban, dabigatran etexilate, dalteparin, enoxaparin, heparin, parnaparin, reviparin, warfarin \\
\hline Antithrombotics: antiplatelets & $\begin{array}{l}\text { abciximab, beraprost, cilostazol, clopidogrel, dipyridamole, epoprostenol, eptifibatide, limaprost } \\
\text { alfadex, nizofenone, ozagrel, sarpogrelate, ticlopidine, tirofiban }\end{array}$ \\
\hline Beta blockers & $\begin{array}{l}\text { acebutolol, alprenolol, amosulalol, arotinolol atenolol, betaxolol, bevantolol, bisoprolol, bopindolol, } \\
\text { bufetolol, carteolol, carvedilol, celiprolol, esmolol, labetalol, landiolol, metoprolol, nadolol, nipradilol, } \\
\text { oxprenolol, penbutolol, pindolol, propranolol, sotalol }\end{array}$ \\
\hline Calcium channel blockers & $\begin{array}{l}\text { amlodipine, aranidipine, azelnidipine, barnidipine, benidipine, bepridil, cilnidipine, diltiazem, } \\
\text { efonidipine, felodipine, manidipine, nicardipine, nifedipine, nilvadipine, nisoldipine, nitrendipine, } \\
\text { verapamil }\end{array}$ \\
\hline Cardiac glycosides & deslanoside, digitoxin, digoxin \\
\hline Diuretics & $\begin{array}{l}\text { amiloride, azosemide, benzylhydrochlorothiazide, bumetanide, chlorthalidone, eplerenone, } \\
\text { ethacrynic acid, furosemide, hydrochlorothiazide, indapamide, isosorbide, mefruside, meticrane, } \\
\text { piretanide, potassium canrenoate, spironolactone, tolvaptan, torasemide, torsemide, triamterene, } \\
\text { trichlormethiazide, tripamide }\end{array}$ \\
\hline Niacin & nicotinic \\
\hline Statins & atorvastatin, fluvastatin, pitavastatin, pravastatin, rosuvastatin, simvastatin \\
\hline
\end{tabular}

